\title{
Enfermedad de Paget ósea: presentación poliostótica en una paciente con cáncer de cérvix
}

\section{Paget's bone disease: polyostotic presentation in a patient with cancer of the uterine cervix}

\author{
Héctor Bojórquez-Castillo,' María de Lourdes Trujillo-Aguirre, ${ }^{2}$ \\ AnitaTarco-Virto ${ }^{3}$ y José Luis Paz-Ibarra ${ }^{4}$
}

Bojórquez-Castillo H, Trujillo-Aguirre ML, Tarco-Virto A, PazIbarra JL. Enfermedad de Paget ósea: presentación poliostótica en una paciente con cáncer de cérvix. Rev Soc Peru Med Interna. 2019;32(I):2I-24.

\begin{abstract}
RESUMEN
Mujer de 65 años con antecedentes de neoplasia maligna de cérvix y neuralgia del trigémino presenta una historia de dos años de dolor crónico e intermitente en región lumbar y cadera izquierda. En el seguimiento ambulatorio se le solicitó estudio radiográfico de cadera, en el que se halló una lesión ósea esclerosa en el alerón ilíaco izquierdo y fosfatasa alcalina de 225 UIL, sugerentes de enfermedad de Paget ósea. Fue hospitalizada para estudio de extensión y confirmación por biopsia ósea ante la posibilidad de metástasis ósea. El estudio óseo, la TEM corporal total y la gammagrafía ósea mostraron lesiones similares en el maxilar inferior derecho y la columna vertebral. Se administró bisfosfonatos mejorando de manera importante el dolor óseo.
\end{abstract}

Palabras clave. Fosfatasa alcalina, enfermedad de Paget ósea, dolor óseo, bisfosfonatos.

\section{ABSTRACT}

A 65-year-old female patient with a history of malignant cervical neoplasia and trigeminal neuralgia presents a twoyear history of intermittent chronic pain in the lumbar region and left hip. On the outpatient follow-up, a radiographic study of the hip showed a sclerous bone lesion in the left iliac wing and an alkaline phosphatase activity of 225 U/L, suggestive of Paget's disease of the bone. She was hospitalized for study by bone biopsy because of the possibility of bone metastasis. The bone survey, total body multi-slice spiral CT and bone scintigraphy showed similar lesions in right lower jaw and spine. Bisphosphonates was administered with significantly improving of bone and facial pain

KEYwords. Alkaline phosphatase, Paget's bone disease, bone pain, bisphosphonates.
I. Médico residente de Medicina interna. Hospital Nacional EsSalud Edgardo Rebagliati Martins (HNERM). Universidad Nacional Mayor de San Marcos (UNMSM).

2. Médico residente de Endocrinología HNERM.

3. Médico endocrinóloga. HNERM.

4. Médico endocrinólogo. HNERM, UNMSM

\section{INTRODUCCIÓN}

La enfermedad de Paget ósea (EPO) es un trastorno del metabolismo óseo que ocurre mayormente sobre hueso envejecido y es el segundo en frecuencia después de la osteoporosis, aunque muchas veces es subdiagnosticado. ${ }^{1}$ Este trastorno consiste en el crecimiento óseo desmesurado de áreas locales producto de un proceso desorganizado y aumentado de resorción ósea, el cual sería activado por factores ambientales, como la participación de un tipo de paramixovirus, y genéticos como la mutación de un gen cuya alteración en la porción ubiquitina incrementa la autofagia en el tejido óseo. ${ }^{1-3}$ Suele ser asintomática pero puede haber dolor óseo y deformación, y otros síntomas producto del sobrecrecimiento óseo local que comprometen la calidad de vida. ${ }^{4}$ El tratamiento actual es con bisfosfonatos y está indicado ante la enfermedad activa sintomática y/o para prevenir complicaciones futuras posibles. ${ }^{5}$

Se presenta el caso de una paciente con diagnóstico de EPO con presentación poliostótica con dolor lumbar y de cadera izquierda, además de neuralgia del trigémino como complicación de la afección del maxilar. 


\section{PRESENTACIÓN DEL CASO}

Mujer de 65 años, natural de Lima, con antecedente de neuralgia del trigémino, desde hace nueve años, con pobre respuesta al tratamiento con carbamazepina y gabapentina.

Además, carcinoma epidermoide de cuello uterino diagnosticada en 2002 tratado con radioterapia intracavitaria y radioterapia externa. Desde hace dos años presenta historia de dolor en columna vertebral lumbar y en cadera izquierda de intensidad moderada de comportamiento intermitente y progresivo en reposo empeorando con el movimiento, y que cedía parcialmente con analgésicos. Niega baja ponderal.

En el examen físico se le halló despierta, en buen estado general, buen estado de hidratación y regular estado de nutrición. Piel sin alteraciones relevantes.

Normocéfala, sin lesiones óseas palpables. En cuello no se palpa la glándula tiroides ni adenopatías. No se palpan lesiones en tórax anterior y en examen pulmonar el murmullo vesicular pasaba sin problemas. A nivel del tórax posterior presenta dolor a la digitopresión a nivel de L3-L5. No se palpan lesiones. Los ruidos cardiacos son rítmicos y no se auscultan soplos. El abdomen es blando y depresible, ruidos hidroaéreos presentes sin visceromegalia. A la palpación, impresiona aumento de volumen óseo a nivel de espina ilíaca

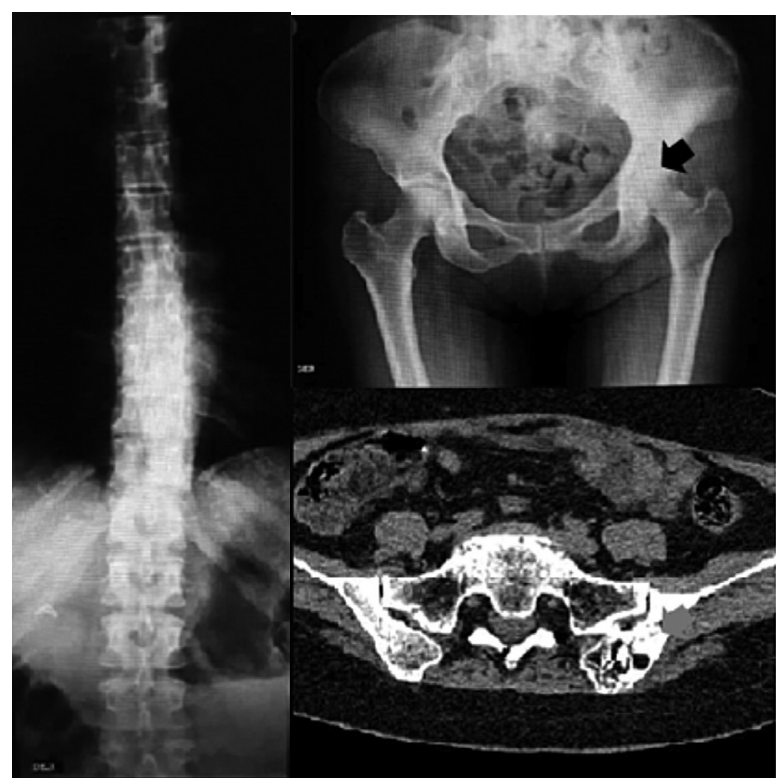

Figura I. Radiografías: lesiones esclerosas en columna vertebral y aleron ilíaco izquierdo. TEM de columna en corte axial mostrando lesión ósea esclerosa del alerón izquierdo.

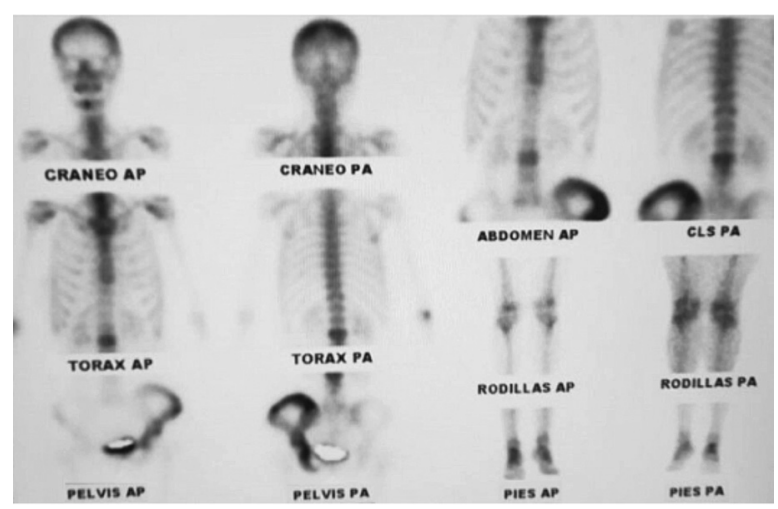

Figura 2. Gammagrafía ósea:áreas de hipercaptación en hemipelvis izquierda y vértebra lumbar, sugestivo de corresponder a Enfermedad de Paget poliostótica.

anterosuperior izquierda, que comparada con la derecha es aproximadamente de 1,05 a 2,0 veces mayor en grosor. No alteración a nivel de articulaciones. A nivel del sistema nervioso central: Glasgow 15, funciones cerebrales conservadas, reflejos osteotendinosos presentes y sin rigidez de nuca.

\section{Exámenes auxiliares}

Hemograma: leucocitos $7700 / \mathrm{mm}^{3}$ con fórmula normal. Plaquetas $415000 / \mathrm{mm}^{3}$.

Hemoglobina $12,3 \mathrm{~g} / \mathrm{dL}$.

Proteína $\mathrm{C}$ reactiva 0,20 ; velocidad de sedimentación globular $20 \mathrm{~mm} / \mathrm{h}$.

Proteínas totales de 7,26 g/dL; albúmina, 4,47 g/dL.

Aspartato aminotransferasa (AST, antes TGO) $31 \mathrm{U} / \mathrm{L}$; alanina aminotransferasa (ALT, antes TGP) ) $24 \mathrm{U} / \mathrm{L}$; bilirrubina total $0,10 \mathrm{mg} / \mathrm{dL}$.

Creatinina $0,49 \mathrm{mg} / \mathrm{dL}$; glucosa $77 \mathrm{mg} / \mathrm{dL}$; urea $24 \mathrm{mg} / \mathrm{dL}$; potasio $4,20 \mathrm{mmol} / \mathrm{L}$; sodio $139 \mathrm{mmol} / \mathrm{L}$; cloro $105 \mathrm{mmol} / \mathrm{L}$; calcio $9 \mathrm{mg} / \mathrm{dL}$; fósforo $4,1 \mathrm{mg} / \mathrm{dL}$; magnesio 2,19 mg/dL. Ácido úrico 2,6 mg/dL; lactato deshidrogenasa 219 U/L (120-246).

Marcadores tumorales: CA-125 11,2 U/mL (0-35); antígeno carcinoembrionario (CEA) $2,42 \mathrm{ng} / \mathrm{mL}$; alfafetoproteína $9,46 \mathrm{ng} / \mathrm{mL}(0-10,9)$; beta-2 microglobulina $1,35 \mathrm{mg} / \mathrm{dL}$. Fosfatasa alcalina $225 \mathrm{U} / \mathrm{L}(45-120)$; gamma-glutamil transpeptidasa (GGTP) $25 \mathrm{U} / \mathrm{L}$ (0-38); osteocalcina $<2 \mathrm{ng} / \mathrm{mL}$ (2-22); parathormona intacta 28,60 pg/mL (11-67); tirotropina (TSH) 3,56 $\mu \mathrm{UI} / \mathrm{mL}$; tiroxina libre (T4L) $0,76 \mathrm{ng} / \mathrm{dL}$.

Estudio radiográfico: lesión esclerótica en el hueso ilíaco izquierdo. 


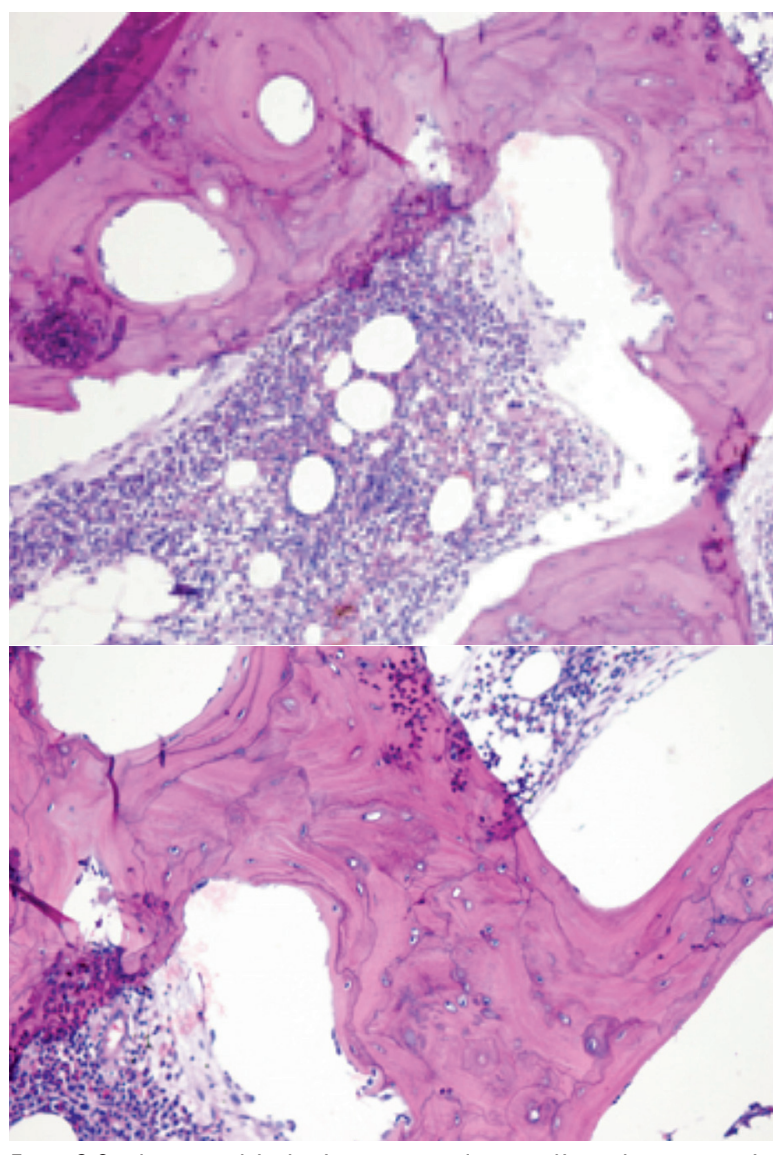

Figura 3. Se observa trabéculas óseas engrosadas y con líneas de cementación irregulares, secundario a proceso remodelativo óseo repetitivo.

Tomografía espiral multicorte (TEM) de columna: lesión ósea esclerosa del alerón izquierdo con trabeculación tosca y aumento volumétrico relativo, aspecto escleroso en los cuerpos vertebrales de L3-L5.

Gammagrafía ósea con Tc-99: se aprecia áreas de hipercaptación a nivel de cráneo: maxilar inferior derecho; a nivel de L3-L5 y a nivel de pelvis: compromiso al nivel del cuerpo del hueso ilíaco y la rama iliopúbica e ilioisquiática izquierda.

\section{Diagnóstico}

El diagnóstico de EPO de presentación poliostótica se plantea ante la presencia de dolor óseo, fosfatasa alcalina elevada y los hallazgos radiológicos múltiples de proceso esclerótico. Se inició el tratamiento con pamidronato, $60 \mathrm{mg}$, endovenoso, y tres semanas después recibió ácido zoledrónico, $5 \mathrm{mg}$, endovenoso (fosfatasa alcalina previa de $133 \mathrm{UI} / \mathrm{L}$ ) con mejoría clínica importante y disminución de los niveles de fosfatasa alcalina (a las 4 semanas posadministración de ácido zoledrónico, 70 UI/L).

\section{DISCUSIÓN}

La EPO, conocida también como osteítis deformante, es una enfermedad del osteoclasto subdiagnosticada, pero considerada en otros países como muy frecuente solo después de la osteoporosis oscilando entre $3 \%$ y $5 \%$ de los pacientes adultos mayores de poblaciones afectadas. ${ }^{6}$ En Perú, los reportes de esta alteración del metabolismo óseo son escasos y se informan como formas clínicas de presentación inusual. ${ }^{7,8}$ Suele afectar a hombres y a mujeres de forma casi indistinta a partir de los 55 años de edad.

A pesar de su reconocida frecuencia, la etiopatogenia no está del todo esclarecida. Se cree que mecanismos genéticos y ambientales jugarían un rol determinante en la aparición. ${ }^{9}$ La mayor cantidad de estudios se centran en la predisposición genética del trastorno, la cual recaería en la mutación del gen del sequestosomal (SQSTM1). Esta mutación afecta al dominio ubiquitina a nivel celular y de esta forma también la autofagia programada en el tejido óseo, lo que permite el sobrecrecimiento desordenado que caracteriza a la enfermedad. ${ }^{3}$

Los síntomas clásicos son dolor óseo y deformidad, y las zonas más comúnmente afectadas el cráneo, la pelvis, la columna vertebral y los huesos largos de miembros inferiores, aunque también la presentación puede ser variopinta. Se ha reportado hipoacusia, estenosis a nivel de medula espinal, síndromes de secuestro vascular, afecciones de pares craneales e, incluso, hidrocefalia. Todas ellas producto de la compresión por el crecimiento óseo desmedido. ${ }^{10}$

El diagnóstico puede tardar en establecerse pues en su mayoría estos pacientes cursan asintomáticos. De sospecharse, un aumento en la fosfatasa alcalina sérica resulta muy útil, y puede corroborarse en radiografías de las áreas comprometidas, con los patrones típicos de la fase esclerótica. Otro tipo de estudios como la gammagrafía ósea, TEM o la tomografía por emisión de positrones (PET-CT) pueden ser útiles principalmente para establecer la extensión de la enfermedad. ${ }^{6,9,11}$ La resonancia magnética con contraste dinámico y la gammagrafía podrían servir para la evaluación resolutiva de las lesiones luego del tratamiento. ${ }^{12,13}$

El diagnóstico diferencial suele establecerse principalmente con lesiones óseas metastásicas, por lo cual la biopsia ósea termina siendo necesaria para su distinción mas no para el diagnóstico usual de la EPO.,11 
El tratamiento de esta entidad busca finalmente detener el crecimiento desmedido de hueso a través del uso de bisfosfonatos como el zolendronato y el pamidronato, para paliar los síntomas y el riesgo de complicaciones tal cual lo establecen las guías. ${ }^{6,9,14}$ Estudios aleatorizados establecen que la normalización de la fosfatasa alcalina no debería ser el objetivo terapéutico por sobre la mejoría clínica. ${ }^{15}$

El cuadro de dolor crónico en nuestra paciente llamó la atención por presentarse con una clara deformidad de la cadera izquierda los cuales junto a un nivel elevado de fosfatasa alcalina (225 UI/L) y un patrón esclerótico típico en el estudio radiográfico de cadera llevó a sospechar de la EPO. Por el antecedente de cáncer de cérvix fue necesario descartar metástasis óseas. Los exámenes séricos no arrojaron alguna peculiaridad más allá de la fosfatasa alcalina elevada sin compromiso de la función hepática, encontrándose los marcadores tumorales dentro de los límites normales. Se amplió el estudio con survey óseo, y gammagrafía ósea encontrando lesiones escleróticas típicas de la osteítis deformante en otras zonas como la columna vertebral y el maxilar inferior derecho (Figuras 1 y 2).

La TEM corporal total sirvió para evidenciar la ausencia de lesiones sugerentes de actividad neoplásica. La biopsia ósea tomada del alerón ilíaco izquierdo mostró hallazgos sugerentes de EPO con trabéculas óseas engrosadas y desordenadas (Figura 3).

El tratamiento se dio con pamidronato, $60 \mathrm{mg}$, endovenoso. Cuando fue reevaluada al mes, refirió disminución en su totalidad del dolor en la columna y la cadera izquierda. Se completó el tratamiento con ácido zoledrónico. El resultado fue la normalización de los niveles de fosfatasa alcalina y la mejoría sintomática del dolor a nivel facial. Este se mantuvo por años resistente al tratamiento con gabapentina y carbamazepina, lo que sugiere que el efecto compresivo del crecimiento óseo a nivel de maxilar inferior derecho fue la causa desencadenante.

\section{REFERENCIAS BIBLIOGRÁFICAS}

I. Alonso N, Calero-Paniagua I, Del Pino-Montes J. Clinical and genetic advances in Paget's disease of bone: a review. Clin Rev Bone Miner Metab. 20I7; I5:37.

2. Teramachi J, Nagata Y, Mohammad K, et al. Measles virus nucleocapsid protein increases osteoblast differentiation in Paget's disease. J Clin Invest. 2016;126:1012.

3. Ralston SH, Langston AL, Reid IR. Pathogenesis and management of Paget's disease of bone. Lancet. 2008;372:I55.

4. Langston AL, Campbell MK, Fraser WD, et al. Clinical determinants of quality of life in Paget's disease of bone. Calcif Tissue Int. 2007;80:I.

5. Singer FR, Bone HG 3rd, Hosking DJ, et al. Paget's disease of bone: an endocrine society clinical practice guideline. J Clin Endocrinol Metab. 20|4;99:4408.

6. Kravets I. Paget's disease of bone: diagnosis and treatment. Am J Med. 2018;|3|:|298-1303.

7. Carrilo-Ñnañez L, Atencia-Matute F, Ortiz-Zegarra C, Hernández-Lopez M. Enfermedad de Paget ósea craneal con hidrocefalia obstructiva y tuberculosis miliar. Rev Soc Peru Med Interna. 20I I;24(I):46-50.

8. Castro F,Angulo J. Osteopatía de Paget. Rev Perú Reum. 1997;3(2):77-3.

9. Singer FR, Bone HG, Hosking DJ, Lyles KW, Murad MH, Reid IR, et al. Paget's disease of bone:An Endocrine Society Clinical Practice Guideline. J Clin Endocrinol Metabolism. 2014;99(I2):4408-22.

10. Ralston SH. Paget's Disease of Bone. N EngI J Med 20I3;368:644-50.

II. Corral Gudino L. Protocolo diagnóstico y tratamiento de la enfermedad de Paget. Medicine. 2016;12:920-4.

12. Libicher M, Kasperk C, Daniels-Wredenhagen M, Heye T, Kauczor HU, et al. Dynamic contrast-enhanced MRI for monitoring bisphosphonate therapy in Paget's disease of bone. Skeletal Radiol. 20I3;42:225-230.

13. Reid IR, Maslowski K. Long-term bone scintigraphy results after intravenous zoledronate in Paget's disease of bone. Calcif Tissue Int. 2017;101:43-49.

14. Appelman-Dijkstra NM, Papapoulos SE. Paget's disease of bone. Best Pract Res Clin Endocrinol Metab. 2018;32(5):657-668.

15. Langston AL, Campbell MK, Fraser WD, MacLennan GS, Selby PL, Ralston SH, for the PRISM Trial Group. Randomized trial of intensive bisphosphonate treatment versus symptomatic management in Paget's disease of bone.J Bone Min Res. 2010;25(I):20-3I.

CORRESPONDENCIA: Héctor Luis Bojórquez Castillo

hlbojorquezc567@gmail.com

FECHA DE RECEPCIÓN: 20 de noviembre de 2018.

FECHA DE ACEPTACIÓN: I5 de enero de 2019. 